\title{
An Agent Based Model to Detect Variations in the Transmission of Potters from Baetica Province
}

\author{
Maria Coto-Sarmiento
}

Patterns of variability found in amphora production can help us understand the mechanism of social learning used in teaching individuals how to make amphorae. For instance, some differences can be detected in the production technique through time and space that might explain different degrees of cultural transmission. ${ }^{1}$ Traditionally, we detect three modes of transmission: vertical, horizontal and oblique. Vertical is when techniques are transmitted from parents to offspring; oblique from individuals of one generation to unrelated individuals of the next generation and horizontal between individuals of the same generation. ${ }^{2}$ In archaeology, cultural transmission has been used for studying the production of handmade pottery. ${ }^{3}$ In our case, we want to know if the modes of transmission could be similar to a more standardized pottery production process typical of the Roman period.

The goal of this study is to analyze the transmission of technical skills among potters within Baetica from the $1^{\text {st }}$ to the $3^{\text {rd }}$ century AD. We propose two questions: first, how amphora production was organized and second, if it would be possible to establish how the production techniques spread from generation to generation. Difficulties in answering these questions are the lack of relevant written sources and uncertainty in the data. To remedy the latter, we use concepts from an evolutionary framework, in order to study the impact that social learning processes may have had on pottery production. Specifically, we want to identify if the differences in the amphorae might reflect differences in the transmission of learning due to the distance between workshops. To do this, we test two hypotheses: 1 . the variability in the morphometric traits is correlated with the geographical distance (closer workshops have more contact) and 2. techniques are only transmitted by vertical/oblique transmission at the beginning (from masters to disciples).

In our study, we analyzed more than 400 samples of olive oil amphorae (Dressel 20) from five different workshops. In each amphora, we measured eight different positions on the rim. We calculated the mean of each measurement for all workshops and the standard deviation of this mean for each workshop. We propose here an Agent Based Model that implements a mechanism of pottery production subject to different social learning processes or cultural transmission modes. Our goal is to quantify which one of these learning processes might explain the distribution observed in our dataset. For the model, we will take into account the two measurements with the highest variation (H and D).

We define five workshops sharing the same production techniques. Each workshop produces amphorae and changes their production techniques by modifying their own techniques VT (Vertical/Oblique Transmission), or copying one from another work- 
shop HT (Horizontal Transmission). We designed three models to test this influence: Model 1 is when VT is the predominant mode of transmission. The probability of copying between workshops is 0 . For instance, workshop A and workshop B produce the amphorae but they do not share techniques because there is no contact between them. Model 2 combines HT and VT $(\mathrm{HT}+\mathrm{HV}(\mathrm{d}))$. The probability of copying is proportional to the geographical distance. For example, two closer workshops, which produce amphorae, will share more similar traits than more remote workshops, like C. In this case, distance will be taken into account in the design of the model. In Model 3, the probability of copying is equal between all workshops. Hence, the amphora workshops will share the same traits in the amphorae production because the distance is not relevant.

For each model, we ran 200 simulations lasting 30,000 time steps. At the end of the model we measured the variation between all the workshops for our two measurements of interest as we did with the empirical data. The results can be seen in Fig. 1. The vertical red line represents the variation measured in the real dataset. The largest variation was located in the Model 1 (VT) instead of Model $3(\mathrm{VT}+\mathrm{HT})$ where the variation is lower than our dataset. By contrast, Model 2 (VT+HT (d)) shows similarity with our dataset (red line).

As preliminary results, we can identify differences among workshops using the Agent Based Model. In the case of the model where VT dominated, the variation was higher: the techniques diverged more due to random drift. By contrast, when HT

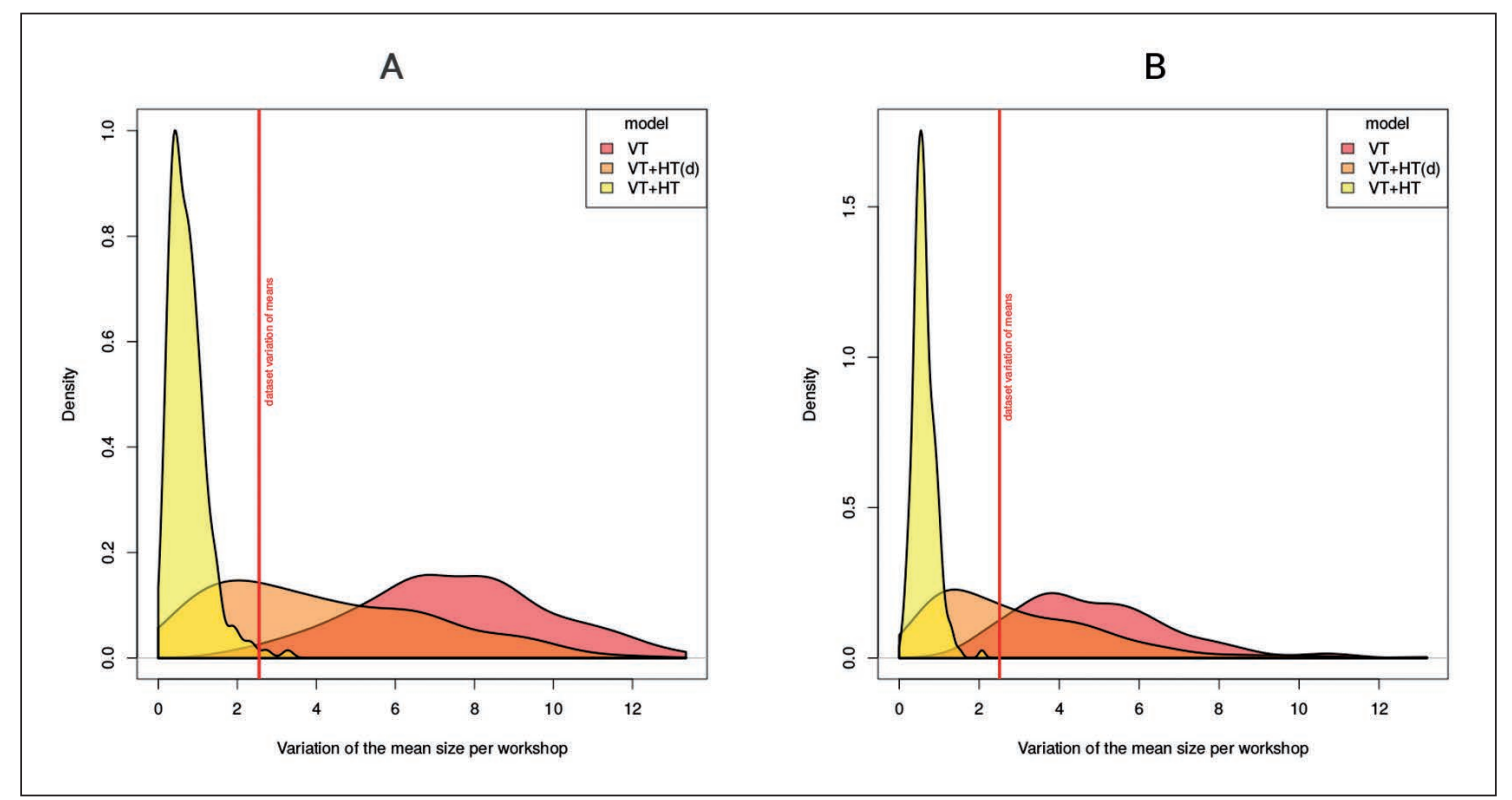

Fig. 1: Results obtained by simulation is given in VT (Model 1), VT+HT (d) (Model 2) and $\mathrm{VT}+\mathrm{HT}$ (Model 3). Red line correspond to the variation of the results of the mean in our dataset. Image $A$ is the measurement $D$ whereas Image $B$ is the measurement $\mathrm{H}$. 
was not correlated with spatial distance, the variation was lower because all the workshops tended to use the same techniques to produce amphorae. The dataset corresponds most to Model 2 when the distance is correlated. The result of Model 2 (VT+HT(d)) shows that by simply adding a cost to social interaction proportional to the distance between workshops allows for reproducing a variability close to the variability observed in the dataset.

Empirical studies and the Agent Based Model show that variability is correlated with geographical distance: the similarity of the traits increases or decreases depending on the geographical distance among the workshops. The results suggest that the similarity in morphological traits are produced by continuous contact between the closest workshops, as is the case in Model 2. This could be explained by the fact that pottery techniques were learned from master to disciple at the beginning, and these potters were exchanging their techniques with the nearest workshops. By using the model presented here, a strong baseline can be set for the exploration of transmission processes related to large-scale production.

\section{Notes}

${ }^{1}$ Eerkens - Lipo 2005.

${ }^{2}$ Cavalli-Sforza - Feldman 1981; Acerbi - Parisi 2006.

${ }^{3}$ Neiman 1995.

\section{Image Credits}

Image by the author.

\section{References}

Acerbi - Parisi 2006

A. Acerbi - D. Parisi, Cultural Transmission Between and Within Generations, Journal of Artificial Societies and Social Simulation 9, 2006, 9.

\section{Berni 2008}

P. Berni, Epigrafía anfórica de la Bética: nuevas perspectivas de análisis, Colección Instrumenta 29 (Universitat de Barcelona).

\section{Cavalli-Sforza - Feldman 1981}

L. L. Cavalli-Sforza - M. W. Feldman, Cultural Transmission and Evolution. A Quantitative Approach (Princeton 1981). 


\section{Eerkens - Lipo 2005}

J. Eerkens - C. Lipo, Cultural Transmission, Copying Errors, and the Generation of Variation in Material Culture and the Archaeological Record, Journal of Anthropological Archaeology 24, 2005, 316-334.

\section{Neiman 1995}

F. D. Neiman, Stylistic Variation in Evolutionary Perspective: Inferences from Decorative Diversity and Interassemblage Distance in Illinois Woodland Ceramic Assemblages, American Antiquity 60, 1995, 7-36.

\section{Remesal 1998}

J. Remesal, Baetican Olive Oil and the Roman Economy, JRA Suppl. 29, 1998, 183-200. 